\title{
Divisão de números naturais: Um olhar para o resto
}

\section{Division of natural numbers: a look at the rest}

Article Info:

Article history: Received 2021-03-29 / Accepted 2021-03-29 / Available online 2021-03-30

doi: 10.18540/jcecvl7iss1pp11970-01-09e

\author{
Jairomar de Araújo Sobrinho \\ ORCID: https://orcid.org/0000-0002-8869-6027 \\ Secretaria Municipal de Educação e Cultura de Couto Magalhães (TO), Brasil \\ E-mail: jairo.matematico@gmail.com. \\ Helves Belmiro Silveira \\ ORCID: https://orcid.org/0000-0002-7724-3911 \\ Universidade Federal do Sul e Sudeste do Pará (UNIFESSPA), Brasil \\ E-mail: helves123@hotmail.com. \\ Orlando Dantona Albuquerque \\ ORCID: Https://orcid.org/0000-0001-5003-4813 \\ Instituto Federal do Pará (IFPA), Brasil \\ E-mail: orlando.albuquerque@ifpa.edu.br. \\ Tarciso Binoti Simas \\ ORCID: https://orcid.org/0000-0002-1687-7582 \\ Universidade Federal do Sul e Sudeste do Pará (UNIFESSPA), Brasil \\ E-mail: tarcisobinoti@gmail.com.
}

\section{RESUMO}

O objetivo deste trabalho é demonstrar a importância da análise dos dados obtidos no algoritmo da divisão, para que haja uma interpretação correta, principalmente no que se refere ao resto dessa divisão. Propõe-se que investigue o mesmo no processo da divisão de números naturais com o objetivo de aprofundar o seu conceito. Foca-se também a relevância e o significado dos demais tratados no tema. Para tanto, foi realizada uma revisão bibliográfica e pesquisa de campo com alunos do Ensino Fundamental da Escola Municipal Cordulina Costa Rêgo da cidade de Couto Magalhães, Tocantins. A pesquisa de campo foi realizada por meio de questionários aplicados aos alunos, a fim de captar informações sobre a temática em questão. Esta pesquisa buscou identificar os diferentes tipos de interpretações que os demais podem dar ao resultado obtido, em diferentes situaçõesproblema que possuem o mesmo algoritmo. Portanto, verificou-se que parte dos alunos tem dificuldade em perceber a existência, não conseguindo interpretar o sentido do resto da divisão, o que impõe a observação de que o professor deve dar a importância que o resto merece, sem dar destaque apenas para o quociente dentro do algoritmo de divisão.

Palavras-chaves: Divisão. Números Naturais. Resto.

\section{ABSTRACT}

The objective of this work is to demonstrate the importance of analyzing the data obtained in the division algorithm, so that there is a correct interpretation, especially with regard to the rest of that division. It is proposed to investigate the same in the process of dividing natural numbers in order to deepen its concept. It also focuses on the relevance and meaning of the other treaties on the theme. To this end, a bibliographic review and field research was carried out with Elementary School students from the Municipal School Cordulina Costa Rêgo in the city of Couto Magalhães, Tocantins. The field research was carried out by means of questionnaires applied to the students, in order to capture information on the subject in question. This research sought to identify the 
different types of interpretations that the others can give to the obtained result, in different problem situations that have the same algorithm. Therefore, it was found that part of the students has difficulty in perceiving the existence, failing to interpret the meaning of the rest of the division, which imposes the observation that the teacher should give the importance that the rest deserves, without giving prominence only to the quotient within the division algorithm.

Keywords: Division. Natural Numbers. Rest.

\section{INTRODUÇÃO}

O presente discorre a respeito da importância do resto no processo da divisão de números naturais. $\mathrm{O}$ conceito de divisão trabalhado no ensino fundamental está relacionado diretamente à operação contrária da multiplicação, podendo ser conceituado com a ideia de partes iguais, ideia de quantas vezes cabe uma determinada quantidade em outra, além da ideia de medidas.

Para Skovsmose (2000), o ensino tradicional de matemática acontece com o professor explicando o conteúdo e, em seguida, os alunos resolvendo algumas atividades. Segundo o autor, desse modo, os alunos aprendem a realizar os algoritmos envolvidos nos problemas, porém, não conseguem compreender a importância dos conceitos envolvidos no processo e não são capazes de estabelecer relações entre os conteúdos e sua aplicabilidade no cotidiano.

Segundo Cássia (2014), é importante sabermos o significado de todos os números que estão envolvidos no algoritmo da divisão. Para a autora, um número muito importante e que quase nunca é lembrado, ou que quase ninguém sabe para o que serve, é o resto da divisão.

Ao realizarmos operações matemáticas dentro do conjunto dos números naturais utilizamos um determinado algoritmo. No entanto, na maioria dos problemas envolvendo divisão de números naturais propostos é solicitado ao aluno que ele dê mais atenção às ideias associadas do que ao quociente da divisão. Sendo assim, observa-se que quase não há uma compreensão por parte dos alunos acerca de todos os conceitos envolvidos na operação. Conforme os Parâmetros Curriculares Nacionais (PCN's):

Embora o estudo dos números e das operações seja um tema importante no currículo do ensino fundamental, constata-se, com frequência, que muitos alunos chegam ao final dessa fase de formação, com um conhecimento insuficiente sobre como eles são utilizados e sem ter desenvolvido uma ampla compreensão dos diferentes significados das operações. (BRASIL, 1998, p. 95).

De acordo com Silva (2020) o resto é conhecido como "o número que sobra na divisão", e quase nunca é atribuída uma importância maior, bem como não é feita uma análise detalhada de seu uso.

Neste sentido, a ideia de um número que sobra é ser chamado de resto, é internalizado na estrutura cognitiva do aluno como algo que não tem importância. E o professor, às vezes, reforça essa ideia, não dialogando com seus alunos acerca do significado que ele possui para a divisão.

Para Sopperlsa (2016), encontramos com facilidade nas avaliações externas, como por exemplo, nas "questões nas provas da Olimpíada Brasileira de Matemática das Escolas Públicas (OBMEP) que envolvem situações cíclicas, isto é, situações que a partir de um dado momento, voltam a se repetir". Como podemos observar na questão a seguir:

Exemplo 1: (OBMEP, 2009) Na sequência 9, 16, 13, 10, 7, ... cada termo, a partir do segundo, é a soma de 7 com o algarismo das unidades do termo anterior. Qual é o $2009^{\circ}$ termo da sequência? 


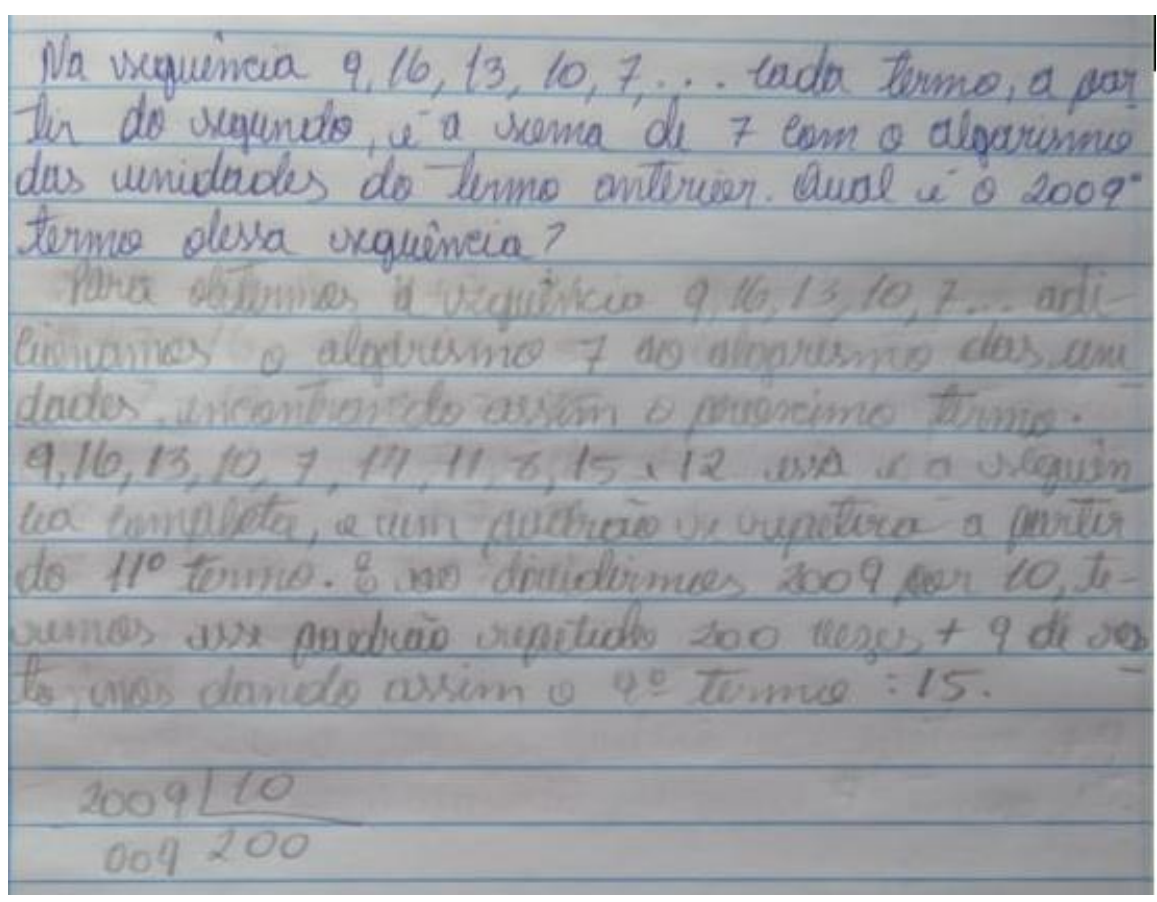

Figura 1 - Resolução do exemplo 1, Acervo Pessoal (2020)

Para solucionar essa questão, o aluno, após encontrar o padrão da sequência, efetuou o algoritmo da divisão para encontrar o resto, pois o mesmo indica em qual termo do padrão da sequência encontra-se a solução para a mesma. Situações assim mostram a importância do resto da divisão, pois em dado evento cíclico, prevê o que acontecerá em um determinado momento futuro.

A escolha do tema justifica-se pela intenção de demonstrar a importância da análise dos dados obtidos no algoritmo da divisão, para que haja uma correta interpretação, em especial do resto dessa divisão, pois como iremos ver no decorrer deste, o resto, dependendo do contexto, pode ter significados diferentes, o que faz com que tenhamos interpretações diferentes para problemas diferentes, influenciando diretamente na solução dos problemas.

Logo, os professores tendem a dar uma maior importância para o quociente de uma divisão, diminuindo a importância que o resto tem e ou pode ter em diversas situações-problema. Neste sentido, tentaremos mostrar o quão importante é o resto no processo da divisão de números naturais, numa tentativa de sensibilizar professores a darem maior importância para este termo dentro do algoritmo da divisão.

O objetivo do trabalho é evidenciar a relevância e o significado para o resto, bem como, identificar os diferentes tipos de interpretações que o ele pode dar ao resultado obtido em situaçõesproblemas distintas que possuem o mesmo algoritmo.

Para alcançar o objetivo proposto, o trabalho analisou periódicos e Trabalhos de Conclusão de Cursos (TCC's), disponíveis em sites que versam sobre a temática. Foi realizada também, uma pesquisa de campo entre os meses de agosto a novembro de 2019, onde foram elaborados e aplicados questionários para alunos do Ensino Fundamental I da Escola Municipal Cordulina Costa Rêgo no município de Couto Magalhães - Tocantins, na tentativa de identificar e entender as dificuldades que os alunos possuem em relação à divisão de números naturais, e em analisar o resto na conjuntura do algoritmo, com vista a minimizar as dificuldades dos alunos em interpretar esses dados.

O trabalho está estruturado da seguinte forma: introdução, discussão teórica, metodologia, resultados encontrados, breve discussões e conclusões.

$\mathrm{Na}$ revisão de literatura, o estudo apoiou-se teoricamente em Skovsmose (2000), Cássia (2014), PCN's (1998), Silva (2020), Ripoll (2015), Marciano (2020), entre outros que discorrem sobre o resto no processo da divisão de números naturais indicados nas referências bibliográficas. 


\section{O RESTO DA DIVISÃO}

objetivando repartir ou medir em partes iguais. No entanto, faz se necessário atentar-se quanto ao significado dos elementos (números) envolvidos no processo da divisão.

De acordo com Marciano (2020), o resto é um dos quatros elementos que compõem o algoritmo da divisão, e esse merece devida atenção. Ao efetuar uma divisão o resto obtido da divisão pode ser nulo ou não. Quando o resto numa divisão é zero, dizemos que essa divisão é exata, caso contrário, chamamos de não exata.

Para Ripoll (2015):

A divisão é, entre as operações básicas, a mais complexa e a que determina maiores desafios para o ensino e para a aprendizagem. Comparada às demais operações elementares, a divisão com números naturais é diferente no seguinte sentido. Enquanto na adição, na subtração e na multiplicação temos dois valores de entrada e obtemos apenas um terceiro valor de saída, que é o resultado da operação, a divisão com naturais envolve dois valores como resultado: o quociente e o resto. $\mathrm{O}$ fato de obtermos duas informações como resultado de uma divisão com naturais faz com que problemas que envolvam esta operação possam ter respostas diversificadas, apesar de um mesmo contexto. (RIPOLL et al, 2015, p. 104).

A fim de fixar esses valores como resultado, o professor pode sugerir que os alunos organizem uma tabela a partir do seguinte problema.

Exemplo 2: Um garoto quer repartir igualmente 31 bolas de gude entre seus 4 primos: o que acontece se ele der 1 bola de gude para cada um dos primos? E se der 2, 3, 4, 5 e 6 ?

Acompanhe a tabela:

Tabela 1 - Resolução do exemplo 2, Acervo Pessoal (2020)

Quantidade de bolas de gude para cada primo

\section{Total de bolas de gude distribuídas}

Total de bolas de gude que sobram

\section{Pode-se dar mais 1 bola de gude para cada primo?}

\begin{tabular}{lccc}
\hline $\mathbf{1}$ & 4 & 27 & Sim \\
\hline $\mathbf{2}$ & 8 & 23 & Sim \\
\hline $\mathbf{3}$ & 12 & 19 & Sim \\
\hline $\mathbf{4}$ & 16 & 15 & Sim \\
\hline $\mathbf{5}$ & 20 & 11 & Sim \\
\hline $\mathbf{6}$ & 24 & 7 & Sim \\
\hline $\mathbf{7}$ & 28 & 3 & Não \\
\hline
\end{tabular}

Desse modo, o total de elementos que sobram, ou seja, o resto deve sempre ser menor que a quantidade para formar novos grupos, ou seja, divisor.

A função do resto em cada situação-problema geralmente não fica bem resolvida, pois faltam discussões mais aprofundadas sobre o assunto.

Nesse caso, a divisão tem o objetivo de descobrir o maior número possível de elementos em cada um dos grupos fixados (o divisor) e também, o total de elementos que sobram (resto), que deve ser menor que o total de grupos fixados.

Segundo Marciano (2020), quando o resto é igual a 0 (zero), a divisão é exata. Logo, o produto do divisor pelo quociente mais o resto é igual ao dividendo, onde o resto tem que ser menor que o divisor.

Sendo assim, podemos perceber que numa divisão temos que: 


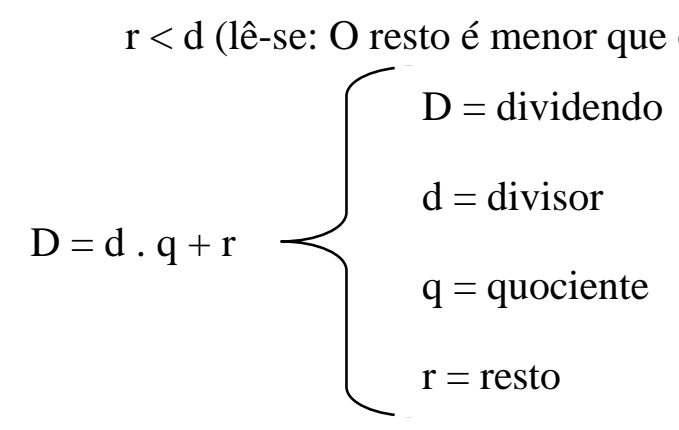

Essa ideia deve ficar clara para os alunos, e, para isso, é necessário que os mesmos vivenciem e discutam situações em sala de aula, a fim de aprofundar seus conhecimentos sobre essa abordagem.

Pelo teorema da divisão Euclidiana temos que a e $\mathrm{b}$ dois números naturais $\operatorname{com} 0<\mathrm{a}<\mathrm{b}$. Existem dois únicos números naturais $\mathrm{q}$ e $\mathrm{r}$ tais que $\mathrm{b}=\mathrm{a} \cdot \mathrm{q}+\mathrm{r}$, com $\mathrm{r}<\mathrm{a}$.

\section{RESULTADOS E DISCUSSÕES}

Todos os alunos que conseguiram resolver a primeira questão do questionário de forma correta tiveram a mesma percepção. Conforme a Figura 2.

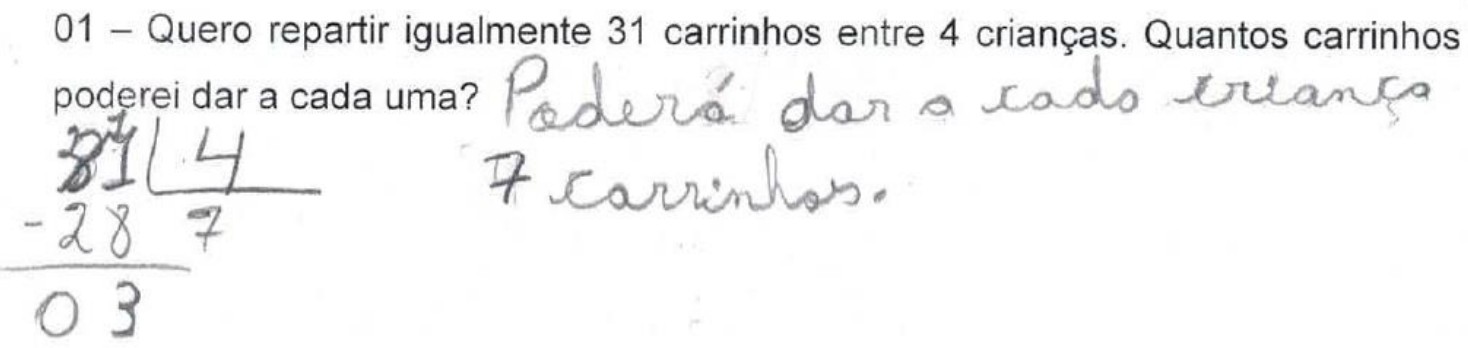

Figura 2 - Questão 1, Acervo Pessoal (2020)

Observamos que, nessa questão, o aluno realiza corretamente o algoritmo da divisão e consegue inferir de forma correta a quantidade de carrinhos que caberia a cada uma das 4 crianças.

Em alguns casos, os alunos até conseguiram ter a noção da sobra de carrinhos. Conforme Figura 3.

01 - Quero repartir igualmente 31 carrinhos entre 4 crianças. Quantos carrinhos
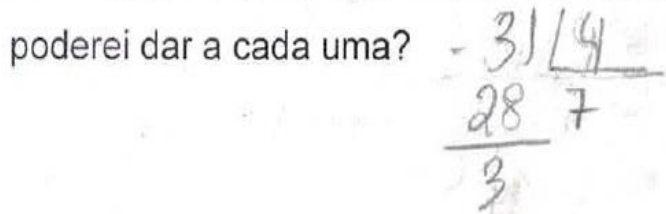

Figura 3 - Questão 1, Acervo Pessoal (2020)

Esse aluno, ao realizar o algoritmo da divisão, observou a existência da sobra de alguns carrinhos, mas ela não influencia na quantidade a ser repartida entre as crianças, já que nesse caso, essa repartição deveria ser igualmente para todas as 4 crianças.

Já na segunda questão, houve divergência de respostas. Conforme Figura 4. 
02 - A Escola Paulo Freire levará 31 alunos para uma excursão. Como a escola não dispõe de ônibus para o transporte, caberá aos professores levarem em seus carros. Cada professor pode levar 4 alunos em seu carro. Quantos carros serão necessários para transportar todos os alunos para a excursão?
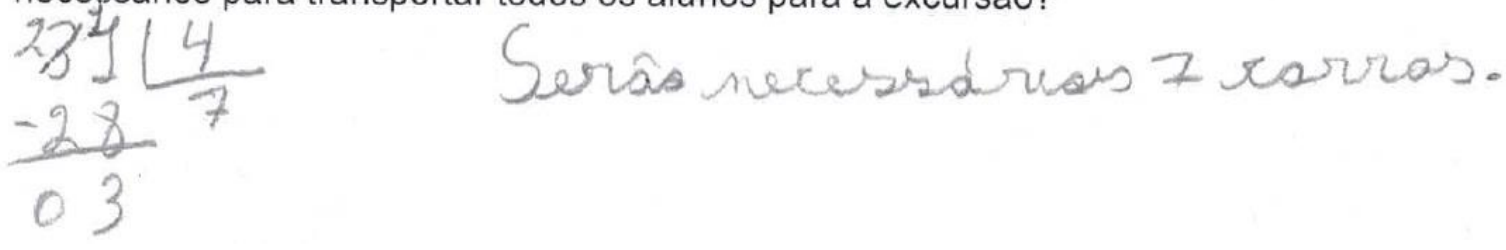

\section{Figura 4 - Questão 2, Acervo Pessoal (2020)}

Esse aluno, apesar de ter realizado o algoritmo da divisão de forma correta, acabou errando, pois levou em consideração apenas o quociente da divisão e não levou em conta o resto não nulo. Conforme Figura 5.

02 - A Escola Paulo Freire levará 31 alunos para uma excursão. Como a escola não dispõe de ônibus para o transporte, caberá aos professores levarem em seus carros. Cada professor pode levar 4 alunos em seu carro. Quantos carros serão necessários para transportar todos os alunos para a excursão?

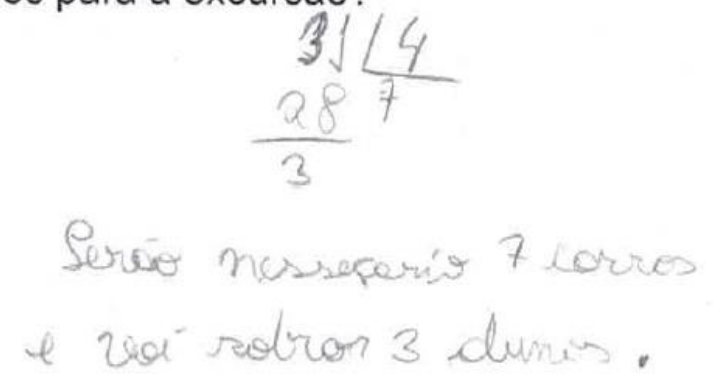

Figura 5 - Questão 2, Acervo Pessoal (2020)

Já esse outro aluno, percebeu que com 7 carros ainda iriam sobrar 3 alunos sem ir a excursão, mas ele não percebeu que havia a necessidade de um oitavo carro para levar esses 3 alunos que sobraram.

Nesse caso, a resposta do problema não será exatamente o resultado da divisão, pois os 7 carros não serão suficientes para levar os 31 alunos.

Já esse aluno conseguiu identificar o total correto de carros que seria necessário. Conforme Figura 6. 
02 - A Escola Paulo Freire levará 31 alunos para uma excursão. Como a escola não dispõe de ônibus para o transporte, caberá aos professores levarem em seus carros. Cada professor pode levar 4 alunos em seu carro. Quantos carros serão necessários para transportar todos os alunos para a excursão?
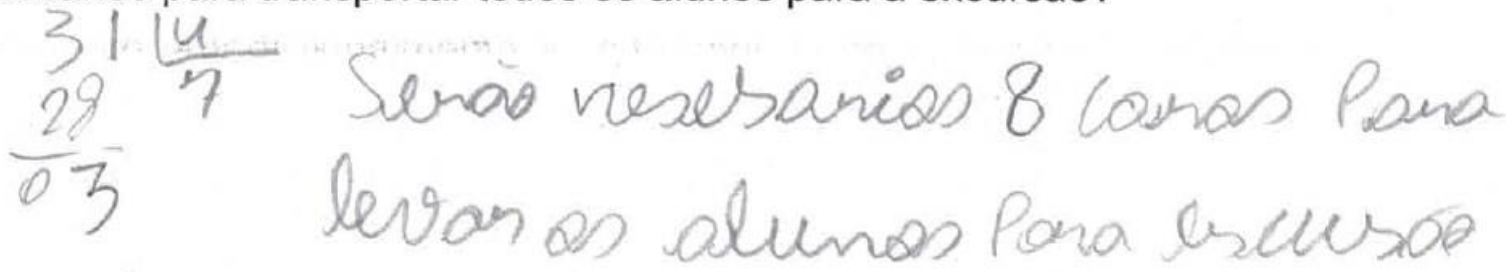

Figura 6 - Questão 2, Acervo Pessoal (2020)

Na Figura 6 é possível perceber que o aluno conseguiu inferir de forma correta a partir da realização do algoritmo da divisão há necessidade do oitavo carro para carregar os 31 alunos para a excursão.

E na terceira questão como cada pizza que sobrou é uma grandeza de natureza contínua, ou seja, é possível ser subdividida, o resultado da divisão poderia ser expresso por uma fração na forma mista: $7 \frac{3}{4}$. Onde cada escola deveria receber 7 pizzas inteiras e $\frac{3}{4}$ de outra. Conforme Figura 7.

03 - A Pizzaria PittizBurg fez 31 pizzas para repartir igualmente entre as 4 escolas municipais. Como eles querem que o total das pizzas seja destruido, que porção caberá a cada escola?

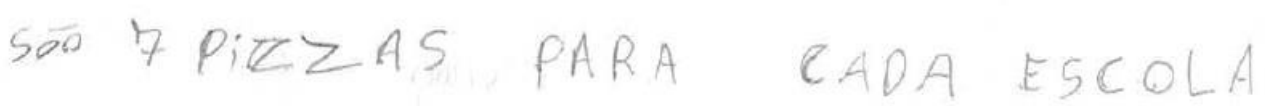

Figura 7 - Questão 3, Acervo Pessoal (2020)

Em todas as três questões, utilizou-se o dividendo 31 e o divisor 4. Essa estratégia foi utilizada propositalmente, na tentativa de mostrar que o resto 3 pode possuir significados diferentes em contextos diferentes, reforçando a necessidade de estratégias de ensino com maior importância para o resto, suas interpretações e significados, na solução de uma situação-problema.

A criança, desde pequena, se depara com situações que as obrigam a trabalhar as ideias de repartir as coisas entre si e também fazer agrupamentos com a mesma quantidade de objetos. Durante as aulas deve-se provocar nos alunos a explorar situações de seu cotidiano em sala de aula. Assim, essas ideias estarão sendo aos pouco sistematizadas, pois, o tempo todo, os alunos se deparam com situações ligadas às discussões cotidianas. 


\section{CONCLUSÕES}

Este trabalho é o resultado de inquietações que se manifestaram no decorrer da docência como educadores de Matemática. O mesmo teve por finalidade verificar e analisar a importância do resto no processo da divisão de números naturais e o modo que o mesmo pode contribuir de forma significativa para o ensino da matemática com base na divisão de números naturais.

É importante que o ensino das operações fundamentais da matemática, em especial a divisão, aconteça de forma contextualizada para que o aluno absorva os conhecimentos matemáticos e que os mesmos façam sentido em suas práticas diárias, conectando os novos conhecimentos a conhecimentos prévios deles, contribuindo assim para uma aprendizagem significativa. E neste sentido, para solucionarmos os problemas encontrados, estratégias de ensino diferenciadas oriundas de uma capacitação adequada podem possibilitar ao professor proporcionar ao aluno a interpretação adequada para o resto, não dando destaque apenas ao quociente, dentro do algoritmo da divisão.

Mostramos, neste trabalho, exemplos em que o mesmo resto pode ter interpretações e significados diferentes, em contextos diferentes. Cabe ao professor, utilizar em suas aulas contextos semelhantes. Fica como sugestão de uma futura pesquisa ao leitor, a produção de um trabalho mostrando mais exemplos contextualizados sobre o resto da divisão de números naturais. $\mathrm{O}$ professor pode, ainda, fazer uso de jogos e ou materiais lúdicos e/ou análise de problemas diários. Fica aqui também como sugestão de pesquisa para o leitor.

No caso da divisão dos números naturais essa contextualização deve ter significado matemático para que a criança adquira competência e lhe possibilite compreender e interpretar as situações problema apresentadas.

Por meio do questionário aplicado, foi possível perceber ainda que a maioria dos alunos não dá significado ao resto da divisão, pois eles não compreendem cada termo dessa operação e acabam interpretando-o apenas como um "número que sobra". Grande parte dos alunos não consegue perceber a importância do resto na divisão de números naturais, sendo que o mesmo faz parte da solução de algumas situações-problema.

Dessa forma, acentua-se que os objetivos desse trabalho foram alcançados a partir da metodologia aplicada aos alunos com uma participação efetiva dos envolvidos, os quais tiveram disposição para colaborar e participar com a aplicação do questionário e assim com a aprendizagem dos mesmos. A metodologia aplicada foi diferenciada buscando contextualizar com o cotidiano do aluno um ensino e aprendizagem da divisão com números naturais.

Espera-se que este trabalho possa contribuir para a relevância da reflexão contínua sobre o fazer docente, o material utilizado e a abordagem dos diversos conteúdos matemáticos no $5^{\circ}$ ano do Ensino Fundamental. Portanto, pode-se dizer que nossa experiência complementa o estudo da divisão na educação básica.

\section{REFERÊNCIAS}

Ausubel, D. P., Novak, J.D., Hanesian, H. (1978). Educational psychology: a cognitive view. 2 ed. NovaYork: Holt, Rinehart and Winston.

BRASIL. Ministério da Educação. Secretaria de Educação Fundamental. (1998). Parâmetros Curriculares Nacionais: Matemática. Brasília: MEC/SEF.

Cássia, A. Para que serve o resto da divisão? In: Estudo Kids. (2020). Disponível em: https://www.estudokids.com.br/para-que-serve-o-resto-da-divisao/ . Acesso em $10 \mathrm{de}$ setembro de 2020.

Gil, A.C. Métodos e técnicas de pesquisa social. (1994). 4 ed. São Paulo: Atlas.

Marciano, E. Para que serve o resto da divisão? In: Escola Educação. (2020). Disponível em: https://escolaeducacao.com.br/para-que-serve-o-resto-da-divisao. Acesso on-line em $06 \mathrm{de}$ setembro de 2020. 
OBMEP. Provas e Soluções. (2020). Disponível em <http://www.obmep.org.br/provas.htm> Acesso on-line em 15 de setembro de 2020.

Ripoll, C. et al. Livro do Professor de Matemática: números naturais. (2015). Rio de Janeiro: SBM.

Silva, L. P. M. Resto da divisão. In: Mundo Educação. (2020). Disponível em: https://mundoeducacao.uol.com.br/matematica/resto-divisao.htm. Acesso on-line em 05 de setembro de 2020.

Skovsmose, O. Cenários para investigação. (2000). Boletim de Educação Matemática. Rio Claro, $\mathrm{n}^{\circ} 14$, p. 66-91.

Soppelsa, J. J. C. Divisão euclidiana: um olhar para o resto. (2016). Diponível em: http://www.ebrapem2016.ufpr.br/wp- content/uploads/2016/04/gd2_Janete_Soppelsa.pdf. Acesso em: 12 de dezembro de 2020. 